\title{
Witness and Counterexample Automata for ACTL
}

\author{
Robert Meolic ${ }^{1}$, Alessandro Fantechi ${ }^{2}$, and Stefania Gnesi ${ }^{3}$ \\ 1 Faculty of Electrical Engineering and Computer Science \\ University of Maribor, Maribor, Slovenia \\ meolic@uni-mb.si \\ 2 Dipartimento di Sistemi e Informatica \\ Università degli Studi di Firenze, Firenze, Italy \\ fantechi@dsi.unifi.it \\ 3 ISTI-CNR, Pisa, Italy \\ gnesi@isti.cnr.it
}

\begin{abstract}
Witnesses and counterexamples produced by model checkers provide a very useful source of diagnostic information. They are usually returned in the form of a single computation path along the model of the system. However, a single computation path is not enough to explain all reasons of a validity or a failure. Our work in this area is motivated by the application of action-based model checking algorithms to the test case generation for models formally specified with a CCS-like process algebra. There, only linear and finite witnesses and counterexamples are useful and for the given formula and model an efficient representation of the set of witnesses (counterexamples) explaining all reasons of validity (failure) is needed. This paper identifies a fragment of action computation tree logic (ACTL) that can be handled in this way. Moreover, a suitable form of witnesses and counterexamples is proposed and witness and counterexample automata are introduced, which are finite automata recognizing them. An algorithm for generating such automata is given.
\end{abstract}

\section{Introduction}

Witnesses that show why a formula is satisfied and (more often) counterexamples that show why it is not satisfied over a model have been used as useful diagnostic information since the first applications of model checking technology. They are usually returned by model checkers in the form of a computation path. However, only for certain kinds of formulae a computation path is able to explain completely the reason of satisfaction or missed satisfaction. Only recently, a greater interest was raised on the study of the relations between the formulae and their counterexamples, on one side looking for richer forms, such as tree-like counterexamples [4] or proof-like counterexamples [10], on the other side establishing the subsets of the logics whose formulae guarantee linear computation paths as counterexamples which completely explain the failure [1, 12].

Our work in this field has been motivated by another trend that has consolidated in the recent years, that is the usage of counterexamples as an help 
to generate test cases $[6,7,8,11,14,15]$. When testing or simulation does not reach an adequate level of coverage (defined by some code coverage metrics, such as statement coverage and branch coverage) new test cases have to be defined, but the process of manually producing test cases for "corner-case" scenarios is time consuming and error prone. Model checking and counterexamples can help: if we have a model of the system, and we model-check on it a formula expressing "there is no uncovered point", a counterexample returns a computation path with enough information to generate the proper test case. In [7] it is shown that in the adoption of this principle with a "conquer and divide" approach in order to attack the typical state space explosion problem, a more effective option is to have the model checker generating not a single counterexample, but all the counterexamples for the given formula. We refer to [7] for more details, while here we focus on the problem of the efficient representation and generation of the set of counterexamples of a given formula.

Action computation tree logic $\left(\mathrm{ACTL}^{1}\right)$ [13] is an action-based version of the branching time temporal logic CTL [3]. ACTL is suitable to express properties of reactive systems whose behaviour is characterized by the actions they perform and whose semantics is defined by means of LTS's. ACTL is adequate with respect to strong bisimulation equivalence, this means that if $p \sim q$, then $p$ and $q$ satisfy the same set of ACTL formulae. To define ACTL an auxiliary logic of actions is introduced. We limit our study to a subset of ACTL that guarantees linear witnesses and counterexamples; we address both witnesses and counterexamples, since one can switch between them using negated formulae. Moreover, we observe that for the purpose of practical applications (e.g. test case discovery) only finite linear witnesses and counterexamples are interesting. Further, we prove that the set of the desired finite linear witnesses and finite linear counterexamples forms a regular language and therefore they can be represented as automata, that will be called witness automaton and counterexample automaton, respectively.

Formal definitions are given in Section 2. In Section 3, we introduce a viable classes of witnesses and counterexamples for application in the field of test case generation and define witness and counterexample automata, such that the sets of witnesses and counterexamples recognized by them form such a viable class. In Section 4 an algorithm to generate witness and counterexample automata is reported and comprehensively explained on examples. Section 5 discusses complexity, implementation, and several directions of possible extension of our work. In Appendix, we show some additional examples of generated automata.

\section{Definitions}

Definition 1. (Labelled transition system)

A labelled transition system (LTS) is a quadruple $\mathcal{M}=\left(S, A c t, D, s_{0}\right)$, where $S$

${ }^{1}$ The acronym ACTL is also used to denote the universal fragment of the CTL, whose original name used in [2] was $\forall \mathrm{CTL}$, later its name has changed for easiness of writing, but generating a conflict with the already used name for Action CTL. 
is a set of states, Act is a set of observable actions (an unobservable action $\tau$ is not in Act), $D \subseteq S \times A c t \cup\{\tau\} \times S$ is the transition relation, and $s_{0} \in S$ is the initial state.

For $A \subseteq A c t$, we let $D_{A}(s)$ denote the set of successors of the state $s$ reachable by an action from the set $A$. Moreover, we let $D_{A}^{\tau}(s)$ denote $D_{A \cup\{\tau\}}(s)$. If $\pi$ is a computation path in an LTS, then $\pi(0)$ is the first state on $\pi$ and $\pi(i+1)$ is the state on the path $\pi$ immediately after the state $\pi(i)$.

\section{Definition 2. (Action formulae)}

The syntax of action formulae over Act is defined by the following grammar where $\chi, \chi^{\prime}$, range over action formulae, and $a \in$ Act:

$$
\chi::=a|\neg \chi| \chi \wedge \chi
$$

The satisfaction of an action formula $\chi$ by an action $a, a \models \chi$, is inductively defined as follows:

$$
\begin{aligned}
& a \models b \quad \text { iff } a=b ; \\
& a \models \neg \chi \quad \text { iff } a \not=\chi ; \\
& a \models \chi \wedge \chi^{\prime} \text { iff } a \models \chi \text { and } a \models \chi^{\prime}
\end{aligned}
$$

We write false for $\alpha \wedge \neg \alpha$, where $\alpha$ is some arbitrarily chosen action, and true stands for $\neg$ false. Moreover, we will write $\chi \vee \chi^{\prime}$ for $\neg\left(\neg \chi \wedge \neg \chi^{\prime}\right)$. An action formula permits the expression of constraints on the actions that can be observed (along a path or after next step); for instance, $a \vee b$ says that the only possible observations are $a$ or $b$, while true stands for "all actions are allowed" and false for "no actions can be observed", that is only silent actions can be performed.

Definition 3. (Action computation tree logic)

The syntax of ACTL is defined by the following grammar, where $\chi, \chi^{\prime}$ range over action formulae, $\exists, \forall$ are path quantifiers, and $\mathbf{X}, \mathbf{U}$ are next and until operators, respectively:

$$
\begin{aligned}
& \varphi::=\text { true }|\neg \varphi| \varphi \wedge \varphi|\exists \gamma| \forall \gamma \\
& \gamma::=\mathbf{X}_{\chi} \varphi\left|\mathbf{X}_{\tau} \varphi\right| \varphi \mathbf{U}_{\chi} \varphi \mid \varphi{ }_{\chi} \mathbf{U}_{\chi^{\prime}} \varphi
\end{aligned}
$$

Let $\kappa(\chi)=\{a|a| \chi\}$. Being interpreted over an LTS $\mathcal{M}=\left(S\right.$, Act, $\left.D, s_{0}\right)$ with total transition relation the satisfaction of a state formula $\varphi$ by a state $s$, $s \models_{\mathcal{M}} \varphi$, and path formula $\gamma$ by a path $\pi, \pi \models_{\mathcal{M}} \gamma$, is inductively defined by:

$s=_{\mathcal{M}}$ true always;

$s=_{\mathcal{M}} \neg \varphi \quad$ iff $s \not \forall_{\mathcal{M}} \varphi$

$s \models \mathcal{M} \varphi \wedge \varphi^{\prime}$ iff $s \models_{\mathcal{M}} \varphi$ and $s \models_{\mathcal{M}} \varphi^{\prime}$;

$s \mid=_{\mathcal{M}} \exists \gamma \quad$ iff there exists a path $\pi$ such that $\pi(0)=s$ and $\pi=_{\mathcal{M}} \gamma$;

$s \models \mathcal{M} \forall \gamma \quad$ iff for all paths $\pi$ such that $\pi(0)=s, \pi \models_{\mathcal{M}} \gamma$;

$\pi \models_{\mathcal{M}} \mathbf{X}_{\chi} \varphi$ iff there exists $\pi(1)$ such that $\pi(1) \in D_{\kappa(\chi)}(\pi(0))$ and $\pi(1) \models_{\mathcal{M}} \varphi$;

$\pi \models_{\mathcal{M}} \mathbf{X}_{\tau} \varphi$ iff there exists $\pi(1)$ such that $\pi(1) \in D_{\{\tau\}}(\pi(0))$ and $\pi(1) \models_{\mathcal{M}} \varphi$; 


$$
\begin{aligned}
\pi \models_{\mathcal{M}} \varphi_{\chi} \mathbf{U} \varphi^{\prime} & \text { iff there exists } i \geq 0 \text { such that } \pi(i) \models_{\mathcal{M}} \varphi^{\prime}, \\
& \text { and for all } 0 \leq j \leq i-1: \pi(j) \models_{\mathcal{M}} \varphi \text { and } \pi(j+1) \in D_{\kappa(\chi)}^{\tau}(\pi(j)) ; \\
\pi \models_{\mathcal{M}} \varphi_{\chi} \mathbf{U}_{\chi^{\prime}} \varphi^{\prime} \text { iff there exists } i \geq 1 \text { such that } & \\
& \pi(0) \models_{\mathcal{M}} \varphi, \pi(i) \models \mathcal{M} \varphi^{\prime}, \pi(i) \in D_{\kappa\left(\chi^{\prime}\right)}(\pi(i-1)), \text { and for all } 1 \leq j \leq i-1: \\
& \pi(j) \models_{\mathcal{M}} \varphi \text { and } \pi(j) \in D_{\kappa(\chi)}^{\tau}(\pi(j-1)) .
\end{aligned}
$$

We write false for $\neg$ true and $\varphi \vee \varphi^{\prime}$ for $\neg\left(\neg \varphi \wedge \neg \varphi^{\prime}\right)$. When the transition system is clear from the context, we write $s \models \varphi$ instead of $s \models_{\mathcal{M}} \varphi$. If $a \models \chi$ and $t=\varphi$ then transition $(s, a, t)$ is called a $(\chi, \varphi)$-transition. Transitions, which are not $(\chi, \varphi)$-transitions, are called $\neg(\chi, \varphi)$-transitions. If $s=\varphi$ we say that $\varphi$ holds in state $s$. An ACTL formula $\varphi$ is satisfied over a given $\operatorname{LTS} \mathcal{M}(\mathcal{M} \models \varphi)$ iff $\varphi$ holds in the initial state of $\mathcal{M}$. The satisfaction of ACTL formulae over LTS $\mathcal{M}_{f}$ having a not total transition relation (i.e. $\mathcal{M}_{f}$ contains deadlocked states) is given as follows: let $\varphi$ be an ACTL formula and $\mathcal{M}_{f}^{\prime}$ be an LTS obtained from $\mathcal{M}_{f}$ by adding $\tau$-loops in all its deadlocked-states; then $\mathcal{M}_{f} \models \varphi$ if $\mathcal{M}_{f}^{\prime} \models \varphi$.

Several useful modalities can be defined, starting from the basic ones:

- $\exists \mathbf{F} \varphi$ for $\exists\left(\right.$ true $\left._{\text {true }} \mathbf{U} \varphi\right)$, and $\forall \mathbf{F} \varphi$ for $\forall\left(\right.$ true $\left._{\text {true }} \mathbf{U} \varphi\right)$ (eventually operators);

$-<\chi>\varphi$ for $\exists \mathbf{X}_{\chi} \varphi$, and $[\chi] \varphi$ for $\neg \exists \mathbf{X}_{\chi} \neg \varphi$ (Hennessy-Milner modalities ${ }^{2}$ );

- $\exists \mathbf{G} \varphi$ for $\neg \forall \mathbf{F} \neg \varphi$, and $\forall \mathbf{G} \varphi$ for $\neg \exists \mathbf{F} \neg \varphi$ (always operators).

Given a model $\mathcal{M}$ and a formula $\varphi$ such that $\mathcal{M} \models \varphi(\mathcal{M} \not \models \varphi)$, a witness (counterexample) is a structure $\mathcal{R}$, in relation with $\mathcal{M}$, that completely shows one of the possible reasons why $\mathcal{M} \models \varphi(\mathcal{M} \not \models \varphi)$. A reason why $\mathcal{M} \models \varphi$ will be called a reason of validity and a reason why $\mathcal{M} \not \models \varphi$ will be called a reason of failure. The type of the relation between $\mathcal{R}$ and $\mathcal{M}$ determines the nature of the witnesses and countererexamples. Linear witnesses and counterexamples are finite or infinite computation paths over $\mathcal{M}$. More complex forms of witnesses and counterexamples [4, 10] are defined as non-linear structures related to the original model $\mathcal{M}$. In our approach, $\mathcal{M}$ is an LTS and $\varphi$ is an ACTL formula. Further, we formally define linear witnesses and counterexamples for an ACTL formula over an LTS; richer forms of witnesses and counterexamples are not directly addressed in this paper.

Definition 4. (Linear witness and counterexample for ACTL formula over LTS) Given an LTS $\mathcal{M}$ and an ACTL formula $\varphi$ such that $\mathcal{M} \models \varphi(\mathcal{M} \not \models)$, a linear witness (counterexample) for $\varphi$ over $\mathcal{M}$ is a sequence of actions that completely shows one of the possible reasons why $\mathcal{M} \models \varphi(\mathcal{M} \not \models \varphi)$.

\section{Witness and Counterexample Automata}

In [4], it has been recognized that for practical end-applications not all witnesses and counterexamples are usable. For example, the whole LTS is always a witness or a counterexample. Following the interpretation of [4], a viable class of witnesses (counterexamples) $\mathcal{V}$ meets the criteria of:

${ }^{2}$ In [13], $\langle\chi\rangle \varphi$ and $[\chi] \varphi$ are defined and used instead as the weak version of the diamond and box operators of Hennessy-Milner logic. 
- Completeness. Every reason of validity (failure), which is important for end-application, can be explained by a witness (counterexample) in $\mathcal{V}$.

- Intelligibility. Witnesses (counterexamples) in $\mathcal{V}$ are specific enough to suit the end-application (e.g. simple enough to be analysed by engineers).

- Effectiveness. There exist effective algorithms for generating and manipulating witnesses (counterexamples) in $\mathcal{V}$.

Note, that these criteria need to be adapted to the end-application. The test case generation approach in [7] is based on finite linear counterexamples, which are no longer than necessary, i.e. they contain only transitions, which contribute to the explanation of a particular reason of failure. Thus, we formally define the viability criteria in the field of test case generation as follows.

Definition 5. (Viability criteria in the field of test case generation)

The class of witnesses (counterexamples) $\mathcal{V}$ is a viable class for application in the field of test case generation iff for the given ACTL formula $\varphi$ and LTS $\mathcal{M}$ there exists a suitable witness (counterexample) in $\mathcal{V}$, which

1. explains all reasons of validity (failure) of $\varphi$ over $\mathcal{M}$ explainable by finite linear witnesses (counterexamples),

2. is as small as possible, and

3. is computable by an effective algorithm.

Let $\mathcal{M}$ be an LTS. In general, all reasons of validity (failure) of an arbitrary ACTL formula $\varphi$ over $\mathcal{M}$ cannot be explained with finite linear witnesses (counterexamples). We avoid the problem of completeness by restricting the approach to the ACTL formulae which guarantee linearity and finiteness, i.e. for which all reasons of validity (failure) can be explained with finite linear witnesses (counterexamples) over all models.

Theorem 1. ACTL formulae of kind $\varphi(\psi)$ as given by the grammar, when satisfied (not satisfied) over an LTS, guarantee linear witnesses (counterexamples):

$$
\begin{aligned}
& \varphi:=\text { true }|\neg \psi| \varphi \vee \varphi \mid \exists\left(\text { true }_{\chi} \mathbf{U} \varphi\right)\left|\exists\left(\operatorname{true}_{\chi} \mathbf{U}_{\chi} \varphi\right)\right| \exists \mathbf{X}_{\chi} \varphi \mid \exists \mathbf{X}_{\tau} \varphi \\
& \psi::=\text { false }|\neg \varphi| \psi \wedge \psi \mid \forall\left(\psi_{\chi} \mathbf{U} \text { true }\right) \mid \forall\left(\psi_{\chi} \mathbf{U}_{\chi} \text { true }\right)\left|\forall \mathbf{X}_{\chi} \psi\right| \forall \mathbf{X}_{\tau} \psi
\end{aligned}
$$

Proof. Theorem 1 can be proved by induction on subformulae. The basic cases are formulae true and false, and every path contains sufficient information for explaining them. Due to a short space, we give a proof only for ACTL formulae of kind $\exists\left(\right.$ true $\left._{\chi} \mathbf{U}_{\chi} \varphi\right)$. Let $\mathcal{M}$ be an LTS, $\gamma=\operatorname{true}_{\chi} \mathbf{U}_{\chi^{\prime}} \varphi^{\prime}, \varphi=\exists \gamma$, and $\mathcal{M}=\varphi$. According to Definition 3, the reason of validity is the existence of paths starting in the initial state of $\mathcal{M}$, which satisfy path formula $\gamma$. Thus, a witness is a proof that a particular path $\pi$ satisfies path formula $\gamma$. If the path $\pi$ itself contains sufficient information to show why $\pi \models \gamma$ then $\pi$ is a linear witness. According to Definition 3 again, for each $\pi \models \gamma$ there exists $i \geq 1$ such that $\pi(i) \models \varphi^{\prime}$ and $\pi(i) \in D_{\kappa\left(\chi^{\prime}\right)}(\pi(i-1))$, and for all $0 \leq j \leq i-1: \pi(j) \in D_{\kappa(\chi)}^{\tau}(\pi(j-1))$. Now, the path from the first state until state $\pi(i)$ needs no additional explanation. We must only show $\pi(i) \models \varphi^{\prime}$. But, due to the induction hypothesis, subformula $\varphi^{\prime}$ guarantees linear witnesses and therefore the suffix of the path from state $\pi(i)$ 
contains sufficient information for explaining $\pi(i) \models \varphi^{\prime}$. Hence, any path $\pi$ such that $\pi \models \gamma$ is a linear witness.

Some formulae with derived modalities can also be used in the presented approach. Indeed, formulae $\exists \mathbf{F} \varphi$ and $\langle\chi\rangle \varphi$ guarantee linear witnesses, while formulae $\forall \mathbf{G} \psi$ and $[\chi] \psi$ guarantee linear counterexamples.

Theorem 1 assures linearity but not finiteness. In fact, all ACTL formulae of the given sublogic, except those of kind $\forall\left(\psi_{\chi} \mathbf{U}_{\chi}\right.$ true $)$, guarantee also finiteness. Therefore, we exclude formulae of this kind from this approach. We also exclude ACTL formulae of kind $\forall\left(\psi_{\chi} \mathbf{U}\right.$ true $)$, as they always hold and have no linear witnesses and no linear counterexamples. For all other kind of formuale of the given sublogic and an LTS $\mathcal{M}$ with total transition relation, we define in a constructive way $\mathcal{V}$-witnesses and $\mathcal{V}$-counterexamples, respectively.

Definition 6. $(\mathcal{V}$-witness and $\mathcal{V}$-counterexample)

(a) A $\mathcal{V}$-witness for ACTL formula true is a path consisting of only one state and no transitions.

(b) A path $\pi$ in $\mathcal{M}$ is a $\mathcal{V}$-witness for ACTL formula $\varphi=\neg \psi$ iff $\pi$ is a $\mathcal{V}$-counterexample for ACTL formula $\psi$.

(c) A path $\pi$ in $\mathcal{M}$ is a $\mathcal{V}$-witness for ACTL formula $\varphi=\varphi_{1} \vee \varphi_{2}$ iff $\pi$ is a $\mathcal{V}$ witness for $\varphi_{1}\left(\varphi_{2}\right)$ and no proper prefix of $\pi$ is a $\mathcal{V}$-witness for $\varphi_{2}$ (resp. $\left.\varphi_{1}\right)$.

(d) A path $\pi$ in $\mathcal{M}$ is a $\mathcal{V}$-witness for ACTL formula $\varphi=\exists\left(\right.$ true $\left._{\chi} \mathbf{U} \varphi^{\prime}\right)$ iff there exists $i \geq 0$ such that suffix of $\pi$ starting in $\pi(i)$ is a $\mathcal{V}$-witness for ACTL formula $\varphi^{\prime}$, and for all $0 \leq j \leq i-1: \pi(j+1) \in D_{\kappa(\chi)}^{\tau}(\pi(j))$ and $\pi(j) \forall_{\mathcal{M}} \varphi^{\prime}$.

(e) A path $\pi$ in $\mathcal{M}$ is a $\mathcal{V}$-witness for ACTL formula $\varphi=\exists\left(\operatorname{true}_{\chi} \mathbf{U}_{\chi^{\prime}} \varphi^{\prime}\right)$ iff there exists $i \geq 1$ such that $\pi(i) \in D_{\kappa\left(\chi^{\prime}\right)}(\pi(i-1))$ and suffix of $\pi$ starting in $\pi(i)$ is a $\mathcal{V}$-witness for ACTL formula $\varphi^{\prime}$, and for all $0 \leq j \leq i-1$ : $\pi(j) \in D_{\kappa(\chi)}^{\tau}(\pi(j-1))$, and also $\pi(j) \notin D_{\kappa\left(\chi^{\prime}\right)}(\pi(j-1))$ or $\pi(j) \forall_{\mathcal{M}} \varphi^{\prime}$.

(f) A path $\pi$ in $\mathcal{M}$ is a $\mathcal{V}$-witness for ACTL formula $\varphi=\exists \mathbf{X}_{\chi} \varphi^{\prime}$ iff $\pi(1) \in$ $D_{\kappa(\chi)}(\pi(0))$ and suffix of $\pi$ starting in $\pi(1)$ is a $\mathcal{V}$-witness for ACTL formula $\varphi^{\prime}$.

(g) A path $\pi$ in $\mathcal{M}$ is a $\mathcal{V}$-witness for ACTL formula $\varphi=\exists \mathbf{X}_{\tau} \varphi^{\prime}$ iff $\pi(1) \in$ $D_{\{\tau\}}(\pi(0))$ and suffix of $\pi$ starting in $\pi(1)$ is a $\mathcal{V}$-witness for ACTL formula $\varphi^{\prime}$.

(h) A $\mathcal{V}$-counterexample for ACTL formula false is a path consisting of only one state and no transitions.

(i) A path $\pi$ in $\mathcal{M}$ is a $\mathcal{V}$-counterexample for ACTL formula $\psi=\neg \varphi$ iff $\pi$ is a $\mathcal{V}$-witness for ACTL formula $\varphi$.

(j) A path $\pi$ in $\mathcal{M}$ is a $\mathcal{V}$-counterexample for ACTL formula $\psi=\psi_{1} \wedge \psi_{2}$ iff $\pi$ is a $\mathcal{V}$-counterexample for $\psi_{1}\left(\psi_{2}\right)$ and no proper prefix of $\pi$ is a $\mathcal{V}$ counterexample for $\psi_{2}$ (resp. $\left.\psi_{1}\right)$.

(k) A path $\pi$ in $\mathcal{M}$ is a $\mathcal{V}$-counterexample for ACTL formula $\psi=\forall \mathbf{X}_{\chi} \psi^{\prime}$ iff $\pi(1) \notin D_{\kappa(\chi)}(\pi(0))$ and $\pi$ contains only two states, or $\pi(1) \in D_{\kappa(\chi)}(\pi(0))$ and suffix of $\pi$ starting in $\pi(1)$ is a $\mathcal{V}$-counterexample for ACTL formula $\varphi^{\prime}$. 
(1) A path $\pi$ in $\mathcal{M}$ is a $\mathcal{V}$-counterexample for ACTL formula $\psi=\forall \mathbf{X}_{\tau} \psi^{\prime}$ iff $\pi(1) \notin D_{\{\tau\}}(\pi(0))$ and $\pi$ contains only two states, or $\pi(1) \in D_{\{\tau\}}(\pi(0))$ and suffix of $\pi$ starting in $\pi(1)$ is a $\mathcal{V}$-counterexample for ACTL formula $\varphi^{\prime}$.

It is straightforward to show that all $\mathcal{V}$-witnesses $(\mathcal{V}$-counterexamples) are finite linear witnesses (counterexamples). The following theorem shows that they are suitable for our approach.

Theorem 2. Let $\pi$ be a finite linear witness (counterexample) explaining a reason of validity (failure) of an ACTL formula $\varphi(\psi)$ over an LTS $\mathcal{M}$. Then, there exists $\mathcal{V}$-witness $(\mathcal{V}$-counterexample), which shows that $\mathcal{M} \models \varphi(\mathcal{M} \vDash \psi)$.

Proof. We claim, that the smallest (not necessary proper and therefore always existing) prefix of $\pi$, which shows that $\mathcal{M} \models \varphi(\mathcal{M} \not \models \psi)$ is a $\mathcal{V}$-witness $(\mathcal{V}$ counterexample). To show this, we observe finite linear witnesses (counterexamples), which are not $\mathcal{V}$-witnesses $(\mathcal{V}$-counterexamples) and prove, that a proper prefix of them exists, which explain $\mathcal{M} \models \varphi(\mathcal{M} \not \models \psi)$. We omit the details of the proof due to the lack of space.

Further, we show that we can characterize not only a single $\mathcal{V}$-witness and $\mathcal{V}$-counterexample, but also the set of all the possible ones. Actually, the number of the possible $\mathcal{V}$-witnesses and $\mathcal{V}$-counterexamples may be infinite and we are interested to a finite representation of them all.

Theorem 3. Let $\mathcal{M}$ be a finite state LTS and $\varphi(\psi)$ an ACTL formula such that $\mathcal{M} \models \varphi(\mathcal{M} \not \models \psi)$. Then, there exists a finite-state automaton which recognizes all $\mathcal{V}$-witnesses $(\mathcal{V}$-counterexamples) for formula $\varphi(\psi)$ over $\mathcal{M}$.

Proof. In order to check whether a path is a $\mathcal{V}$-witness $(\mathcal{V}$-counterexample), we just need to see whether it is a path over $\mathcal{M}$ and if it has the form given in Definition 6 . Since the characterizations given in Definition 6 are given with a single right recursion, they can be expressed as a regular grammar. The language of $\mathcal{V}$-witnesses $(\mathcal{V}$-counterexamples) is the intersection of the regular language recognized by this grammar and the regular language of the finite paths over $\mathcal{M}$. Hence, it is a regular language and can be recognized by a finite state automaton.

Definition 7. (Witness and counterexample automata)

A witness (counterexample) automaton for an LTS $\mathcal{M}$ and an ACTL formula $\varphi$ is an automaton which recognizes the language of all $\mathcal{V}$-witnesses $(\mathcal{V}$-counterexamples) of $\varphi$ over $\mathcal{M}$.

Now, only an effective algorithm for generation of witness and counterexample automata is missing to fit the viability criteria in Definition 5 .

\section{Implementation}

We present here an elegant recursive algorithm that, given a LTS and a formula $\varphi$ from the subset of ACTL given in Theorem 1, generates the witness or counterexample automaton WCA for the formula $\varphi$ over the given LTS. If the formula $\varphi$ holds in the initial state of LTS, the generated WCA is a witness automaton, otherwise, it is a counterexample automaton. The algorithm is 


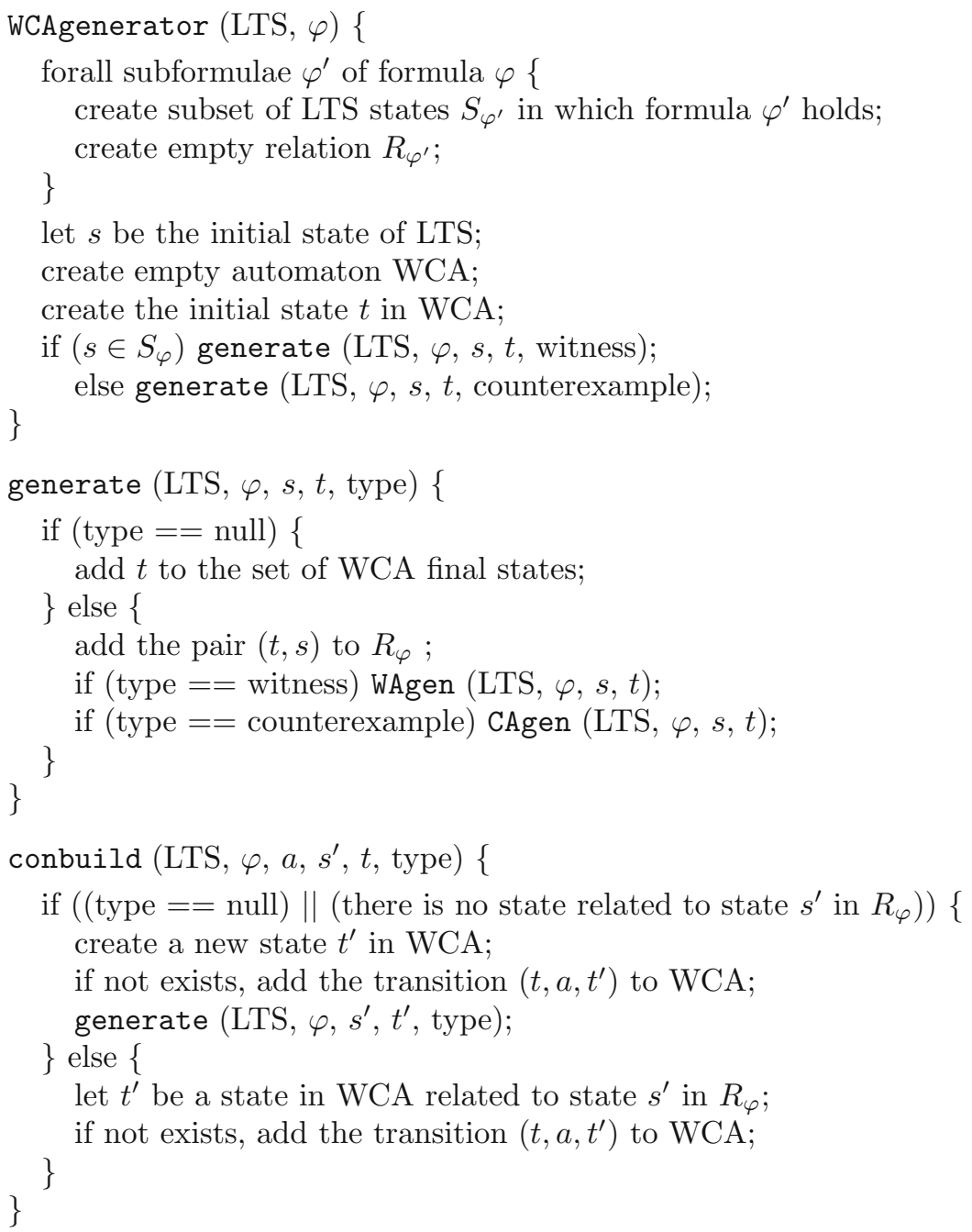

Fig. 1. Main function and two auxiliary functions of the algorithm

a direct implementation of the definitions of $\mathcal{V}$-witnesses and $\mathcal{V}$-counterexamples and it is given in a $\mathrm{C}$-like pseudocode. It consists of the main function $W C A$ generator, two auxiliary functions generate and conbuild (Fig. 1), and functions processing ACTL formulae (Fig.2, 3). For the purpose of further work, the part for generation of counterexample automata is extended with formulae of type $\forall\left(\psi_{\chi} \mathbf{U}_{\chi}\right.$ true $)$. For them, $\mathcal{V}$-counterexamples have not been defined. Computation paths recognized by the obtained automaton for this kind of ACTL formulae explain only those reasons of failure which can be explained with finite linear counterexamples. Due to the lack of space, we are not able to discuss details of this feature. 


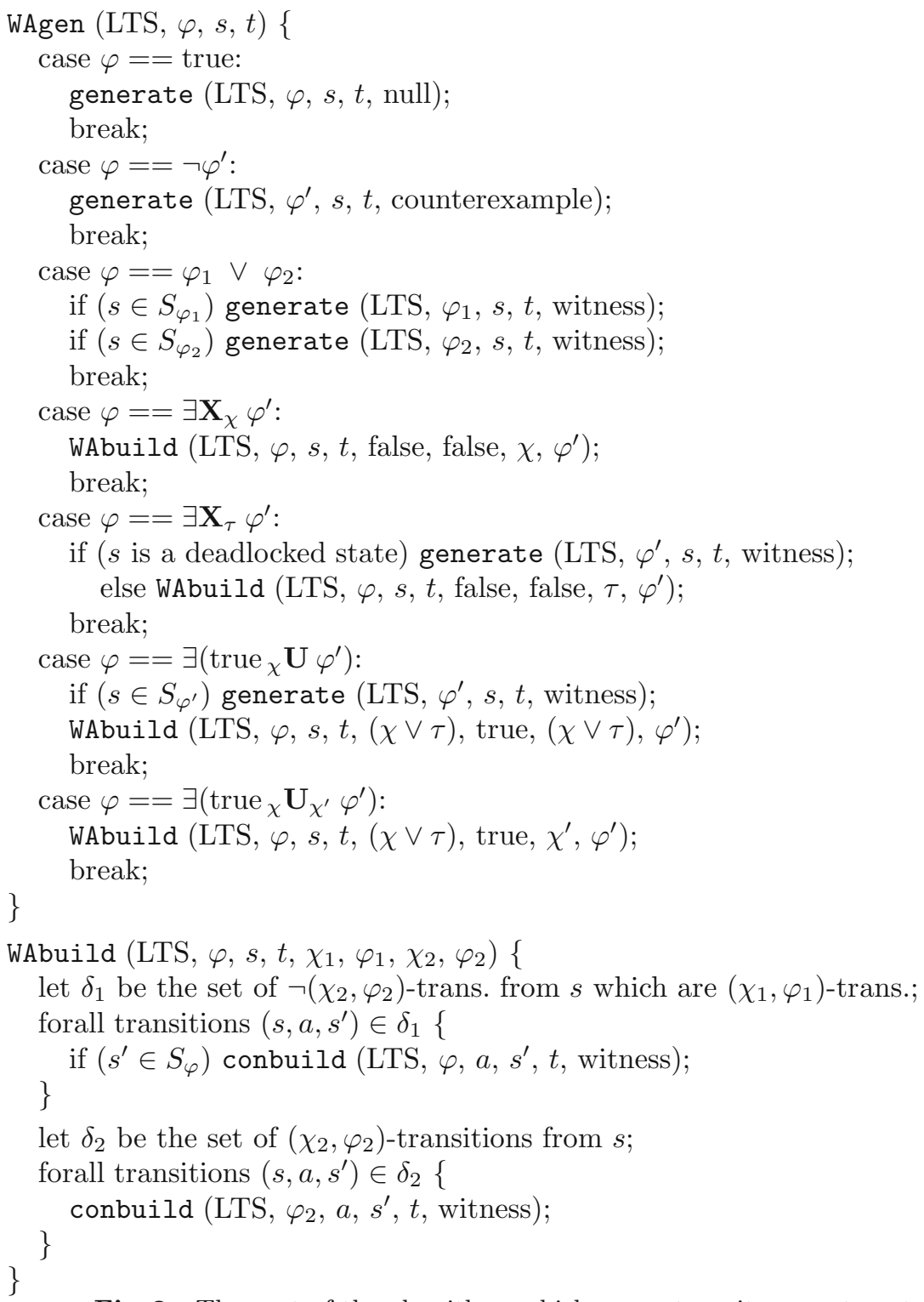

Fig. 2. The part of the algorithm, which generates witness automaton

The algorithm proceeds by visiting a portion of the state space of LTS. The visit is guided by the structural analysis of the formula itself, hence it is terminated when the leaves of the formula are reached. In ACTL, leaves can only be the formula true or the formula false. LTS is unfolded if a sequence of subformulae of $\varphi$ matches with a loop in it. The visit is implemented by a depth-first search by recursion, and hence it has to be remembered which states of LTS have already been visited with a particular subformula of $\varphi$. Unfortunately, the resulting au- 


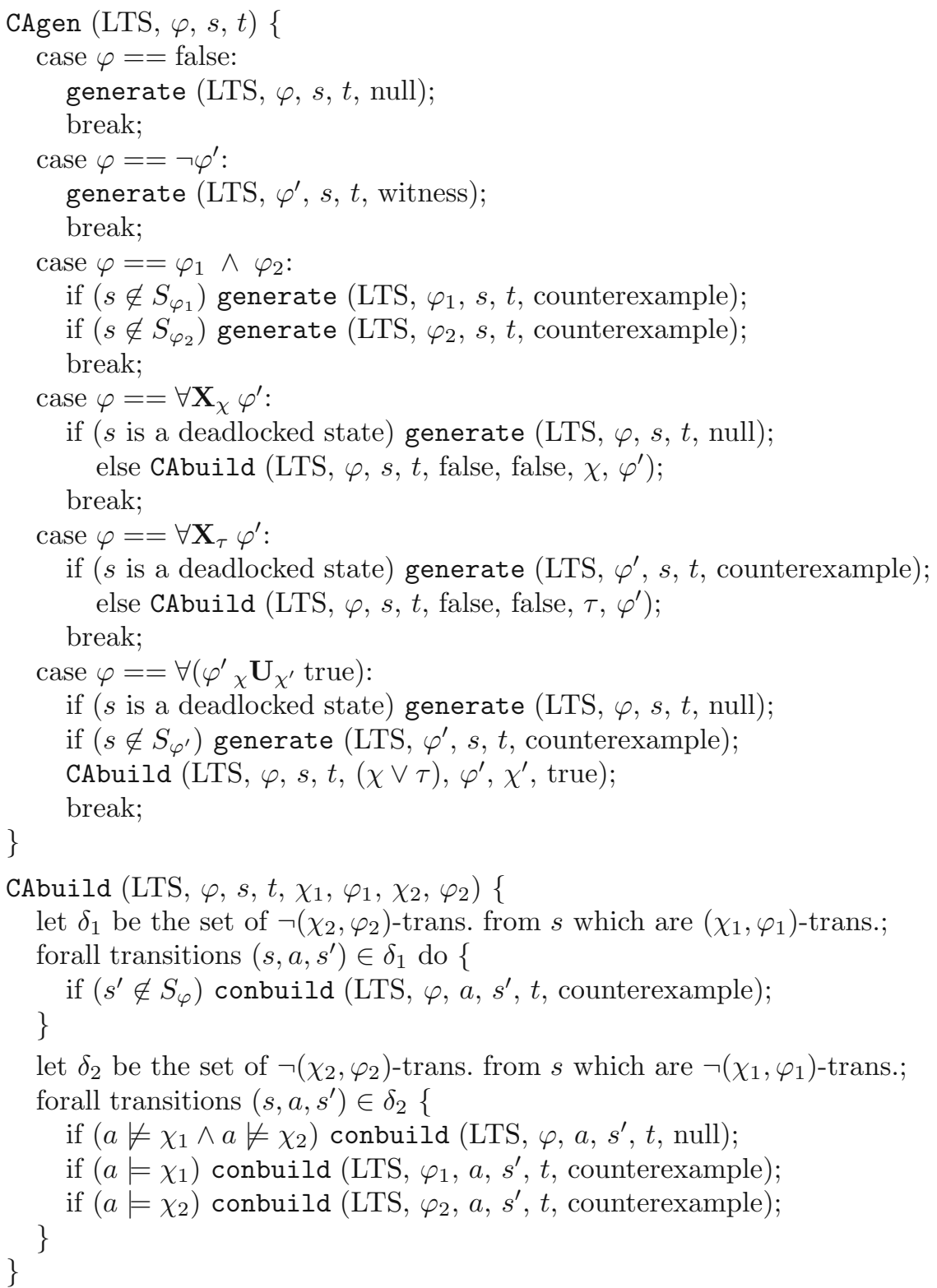

Fig. 3. The part of the algorithm, which generates counterexample automaton

tomaton can be slightly incorrect. It may recognize some paths, which are finite linear witnesses (counterexamples) but not $\mathcal{V}$-witnesses $(\mathcal{V}$-counterexamples). However, it can always be minimized to fit Definition 7 . We will discuss about this in the next section.

The algorithm is further explained in details using a simple LTS and two simple ACTL formulae (Fig. 4). It starts in function WCAgenerator. The first 


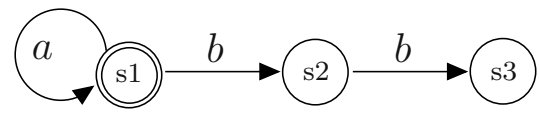

(a) The model: $S=a . S+b . b . n i l$

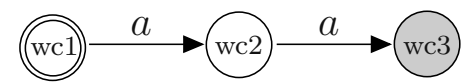

(b) Witness automaton for $\exists \mathbf{X}_{a} \exists \mathbf{X}_{a}$ true

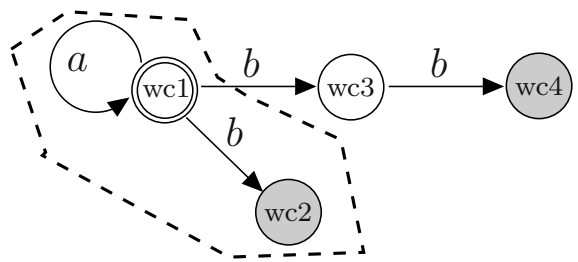

(c) Witness automaton for $\exists \mathbf{F} \exists \mathbf{X}_{b}$ true

Fig. 4. Two examples of generated witness automata. For the second ACTL formula, the resulting automaton must be properly minimized to obtain the correct witness automaton indicated by a dashed polygon

action is actually a call to a model checker, which computes $S$ and initializes $R$ for all subformulae of $\varphi$. $S$ contains for each subformula the subset of states that satisfy the subformula. Thus, the algorithm takes for granted the information about validity of the subformulae on each state. $R$ is a relation implemented as a set of pairs, where for each state of the LTS visited with a particular subformula the related state in WCA is stored. Variables WCA, $S$, and $R$ are global, all others are local. Here is a program trace for the example in Fig. 4b.

ACTL model checking on $\mathrm{S}$

$\operatorname{EX}\{a\} \operatorname{EX}\{a\}$ true $==>$ TRUE

@@ WCAgenerator: created empty witness automaton WC1S

(Q) WCAgenerator: created initial state wc1

@e generate: starting formula 'EX $\{a\} \operatorname{EX}\{a\}$ true' for ( $s=s 1, t=w c 1$ )

QQ generate: added pair (wc1,s1) to $\mathrm{R}$ for the current formula

Q0 WAbuild: chosen transition s1-a->s1 from delta2

Q0 conbuild: created state wc2, created transition wc1-a->wc2

(e) generate: starting formula ' $E X\{a\}$ true' for ( $s=s 1, t=w c 2$ )

(Q6) generate: added pair (wc2,s1) to $\mathrm{R}$ for the current formula

(e) WAbuild: chosen transition $\mathrm{s} 1-\mathrm{a}-\mathrm{s} 1$ from delta2

(e) conbuild: created state wc3, created transition wc2-a->wc3

(e) generate: starting formula 'true' for ( $s=s 1, t=w c 3$ )

QQ generate: added pair (wc3,s1) to $\mathrm{R}$ for the current formula

(Q0) generate: state wc3 marked as final

(@) Witness automaton has been constructed. 
After an ACTL model checker determines that formula $\exists \mathbf{X}_{a} \exists \mathbf{X}_{a}$ true holds in the initial state $\mathrm{s} 1$ of $\mathrm{S}$, a generation of the witness automaton is started. A new automaton WC1S and its initial state wc1 are created. Function generate makes initial states of S and WC1S to be related for the formula $\exists \mathbf{X}_{a} \exists \mathbf{X}_{a}$ true. Then, function WAgen is started. The outermost operator is $\exists \mathbf{X}_{a}$ and therefore function WAbuild is called with parameters $\chi_{1}=$ false, $\varphi_{1}=$ false, $\chi_{2}=a$, and $\varphi_{2}=\exists \mathbf{X}_{a}$ true. Set $\delta_{1}$ is empty, because there is no $\left(\chi_{1}, \varphi_{1}\right)$-transition. Set $\delta_{2}$ contains only the transition $(\mathrm{s} 1, a, \mathrm{~s} 1)$. State $\mathrm{s} 1$ has not been visited with formula $\exists \mathbf{X}_{a}$ true, yet, and therefore there is no state in WC1S related to it. Function conbuild creates a new state wc2 and the transition (wc1, a, wc2) in WC1S. Then, function generate is recursively called for the formula $\exists \mathbf{X}_{a}$ true. In this call, subformula $\varphi_{2}=$ true. Again, set $\delta_{1}$ is empty, while set $\delta_{2}$ contains the transition $(\mathrm{s} 1, a, \mathrm{~s} 1)$. Because state $\mathrm{s} 1$ has not been visited with formula true, yet, function conbuild creates a new state wc3 and transition (wc2, $a$, wс3). Further, state wc3 is marked as final and the recursive calls end.

The usage of relation $R$ is better shown on a program trace for ACTL formula $\exists \mathbf{F} \exists \mathbf{X}_{b}$ true. Actually, this is an abbreviation of formula $\exists$ (true true $_{\mathbf{U}} \exists \mathbf{X}_{b}$ true).

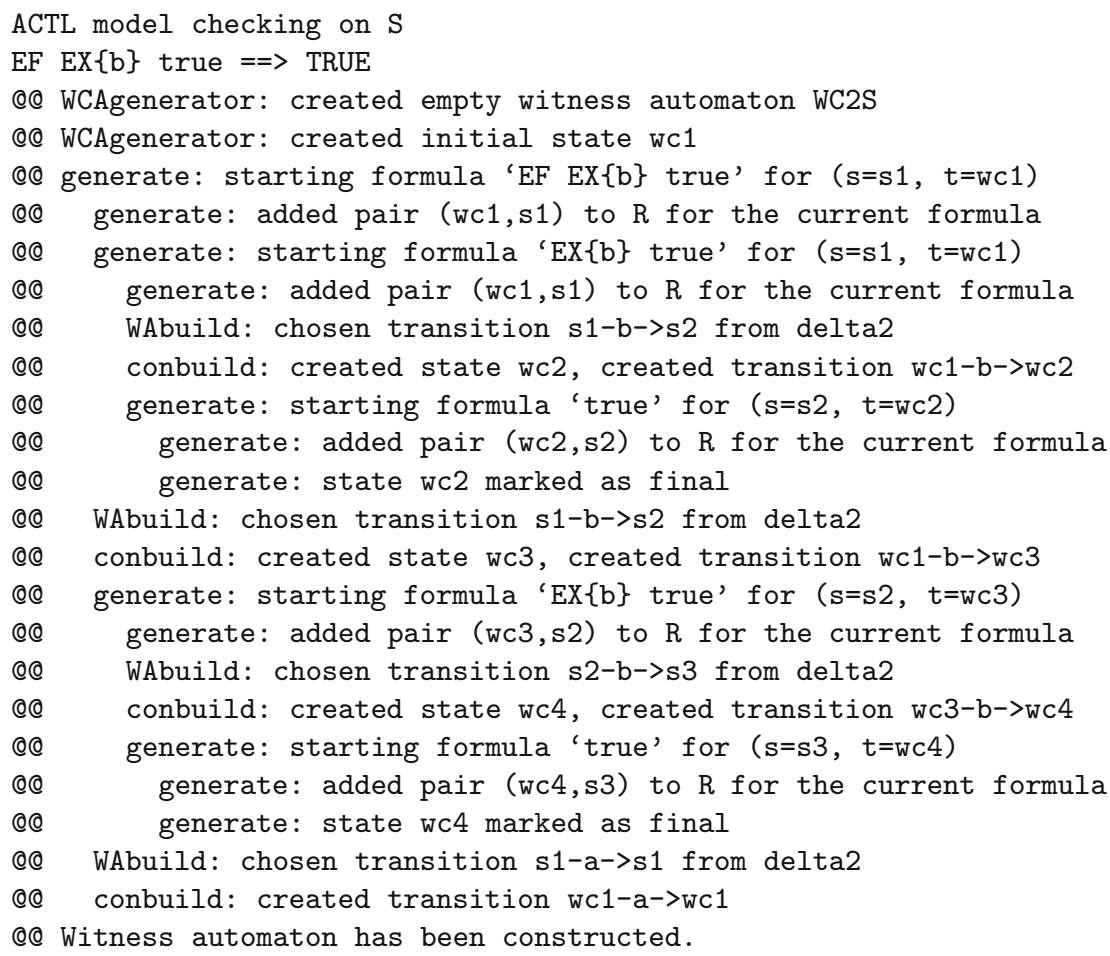

Because subformula $\exists \mathbf{X}_{b}$ true holds in the initial state of $\mathrm{S}$, first a $\mathcal{V}$-witness for it is generated. State wc2 and transition (wc1, b, wc2) are created. Then, function WAgen continues and calls function WAbuild with parameters $\chi_{1}=$ true, $\varphi_{1}=$ true, $\chi_{2}=$ true, and $\varphi_{2}=\exists \mathbf{X}_{b}$ true. Set $\delta_{1}$ is empty, while set $\delta_{2}$ contains 
transitions $(\mathbf{s} 1, a, \mathbf{s} 1)$ and $(\mathbf{s} 1, b, \mathbf{s} 2)$. The transition with action $b$ is chosen first. Formula $\exists \mathbf{X}_{b}$ true has been already visited in state $\mathbf{s} 1$, but not in state s2. Therefore, state wc3 and transition (wc1, b, wc3) are created. Afterwards, the algorithm continues in the state s2. State wc4 and transition (wc3, b, wc4) are created. Because subformula true has been reached, state wc4 is marked as final and the path is finished. Now, function WAbuild must also process transition (s1, $a, \mathrm{~s} 1)$ from $\delta_{2}$. State s1 has been visited with formula $\exists \mathbf{F} \exists \mathbf{X}_{b}$ true before, thus a new state is not created. State $\mathbf{s} 1$ is related to state wc1 in rela-

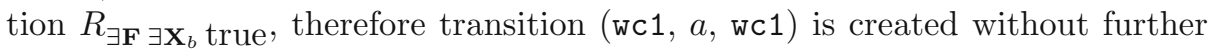
recursive calls.

\section{Discussion}

The algorithm for witness and counterexample automata generation basically works by following the given LTS and using unfolding when necessary, with an unfolding depth of at most the length of the formula. Therefore, the complexity is not higher than the size of the LTS (states and transitions) times the length of the formula. This is exactly the same complexity of an explicit model checking algorithm which has to be employed to compute the labeling of the LTS.

We have implemented the algorithm as an extension of a BDD-based ACTL model checker. Although LTSs are represented by BDDs and BDD-based functions are used for navigating the LTS, the algorithm indirectly still involves an implicit enumeration in functions WAbuild and CAbuild, where transitions are chosen from $\delta_{1}$ and $\delta_{2}$ one by one and then for each next state on the path a new recursive call is made. An open question remains whether a more efficient symbolic algorithm exists.

The last example in the previous section and some of the examples given in Appendix clearly show that the resulting automata may contain redundancy. In fact, there are two different kinds of redundancy. At first, some equal paths may be presented more than once. This is not very disturbing and among other reasons it appears because the program does not identify two semantic equal subformulae as the same one. For example, in the witness automaton generated for the ACTL formula $\left(\exists \mathbf{X}_{\chi} \varphi\right) \vee\left(\exists \mathbf{X}_{\chi} \varphi\right)$, all paths are doubled. The second type of redundancy is much more problematic as it leads to the incorrect result. It appears due to the fact, that during the generation of the automaton the algorithm does not check, whether one of the created paths explaining one reason of validity (failure) is a proper prefix of another created paths explaining another reason of validity (failure). In such case, only the shorter path is a $\mathcal{V}$-witness $(\mathcal{V}$ counterexample) and thus the generated automaton should recognize only the shorter one and not both. The ACTL formulae, which are subject to this kind of redundancy are all those which contain Boolean operator $\vee$ or $\wedge$, explicitely or implicitely as, for example, formula $\exists$ (true $\left.{ }_{\chi} \mathbf{U} \varphi^{\prime}\right)$ and derived formula $\exists \mathbf{F} \varphi$. To obtain the correct automaton, all extra paths must be later eliminated by a proper minimization. 
A result which is related to our work is the definition of more expressive tree-like counterexamples for Kripke Structures and CTL; such counterexamples are used as a support to guide a refinement technique [4]. The main difference with respect to our approach is that a tree-like counterexample is in its entirety a proof that the formula is not satisfied. Our counterexample automaton gives instead the set of linear counterexamples, each of which can be taken separately as a traditional counterexample. An evolution of tree-like counterexamples is represented by proof-like counterexamples [10], used to extract proofs for the non satisfiability of a formula over a model. Closer to our approach is the multiple counterexamples generation of $[5,9]$, which generates all the counterexamples up to a given length, expressed as a single counterexample trace annotated with possible values of binary variables.

There are some possible directions for further work. We have considered only finite witnesses and counterexamples, which are the ones suitable to be used as actual test cases (see [7]). Having more rich notions of acceptance than linear languages could provide the possibility of characterizing sets of more informative witnesses and counterexamples. In order to deal with infinite counterexamples and witnesses the same approach can be followed, for example, using Büchi automata for recognizing a language of infinite words. In this way, if the transition relation is total and if witnesses and counterexamples are extended to become infinite paths, our work becomes adequate to the work presented for CTL in [1].

An interesting extension of the given algorithm would be a generation of non-linear forms of witnesses and counterexamples. The core of the algorithm are functions WAbuild and CAbuild. We implemented them in a more general form w.r.t. what needed in this approach. For example, function WAbuild will also process ACTL formula $\exists\left(\varphi_{1 \chi} \mathbf{U}_{\chi^{\prime}} \varphi_{2}\right)$, where parameter $\varphi_{1}$ is not just a simple formula true or false, although a witness for this formula is not always a linear computation path. The algorithm will produce an automaton recognizing linear witnesses and the main paths (sometimes referred as backbones) of non-linear witnesses. There will be no extra information given about which recognized witness completely explains the validity and which one is only a main path of a non-linear witness. Such general implementation allows extensions. If parameter $\varphi_{1}$ is not a simple formula true or false and if an explanation of validity is added to all states on the main path, we get richer non-linear forms of witnesses. Thus, the given algorithm can serve also as a basis for generation of tree-like witnesses and counterexamples. Note that for the formulae which guarantee linearity and finiteness of witnesses (counterexamples) the presented witness and counterexample automata explain all reasons of validity (failure) over a given model and thus they are equivalent to the tree-like witnesses and counterexamples, respectively.

\section{Conclusions}

We have defined witness and counterexample automata, which are intended to be used in the field of test case generation. These automata recognize $\mathcal{V}$-witnesses 
and $\mathcal{V}$-counterexamples which are finite linear witnesses and counterexamples for a given formula over a given LTS. The main result of the paper is the algorithm for generating witness and counterexample automata for a given LTS and a given ACTL formula from a subset of ACTL formulae which guarantee finite linear witnesses and counterexamples. The algorithm has been implemented and a stand-alone demo application has been made available online on http://fmt.isti.cnr.it/WCA/.

It seems reasonable that the given approach works as well with a state based formalism (such as Kripke structures) and a state based temporal logic (such as CTL). This needs to be verified: the very definition of witnesses, counterexamples, and automata recognizing them is actually highly sensitive to the logic used and to the assumptions on the models.

\section{Acknowledgements}

This work has been partially supported by Italian MIUR PRIN 2002 COVER project. The first author has been partially supported by a grant from Government of Italy through Italian Cultural Institute in Slovenia. We wish to thank Gianluca Trentanni for his help in the realization of the on-line demo application.

\section{References}

[1] F. Buccafurri, T. Eiter, G. Gottlob, N. Leone. On ACTL formulas having linear counterexamples. Journal of computer and syst. sciences, 62(3), 2001, pp. 463515. 259, 272

[2] E. M. Clarke, O. Grumberg, D. E. Long. Model Checking and Abstraction ACM Transaction on Programming Languages and Systems, (16)5, 1994, pp. 1512-1542. 260

[3] E. M. Clarke, E. A. Emerson, A. P. Sistla. Automatic Verification of Finite State Concurrent Systems using Temporal Logic Specifications. ACM Transaction on Programming Languages and Systems, 8(2), 1986, pp. 244-263. 260

[4] E. M. Clarke, S. Jha, Y. Lu, H. Veith. Tree-like Counterexamples in Model Checking. In 17th IEEE Symp. on Logic in Computer Science (LICS), 2002, pp. 19-29. 259, 262, 272

[5] F. Copty, A. Irron, O. Weissberg, N. Kropp, G. Kamhi. Efficient Debugging in a Formal Verification Environment. In Conf. On Correct Hardware Design and Verification Methods (CHARME), LNCS 2144, 2001, pp. 275-292. 272

[6] A. Časar, Z. Brezočnik, T. Kapus. Exploiting Symbolic Model Checking for Sensing Stuck-at Faults in Digital Circuits. Informacije MIDEM, 32(3), 2002, pp. 171-180. 260

[7] A. Fantechi, S. Gnesi, A. Maggiore. Enhancing test coverage by back-tracing model-checker counterexamples. In Int. Workshop on Test and Analysis of Component Based Syst. (TACOS), 2004, to appear in Electronic Notes in Theoretical Computer Science. 260, 263, 272

[8] D. Geist, M. Farkas, A. Landver, Y. Lichenstein, S. Ur, Y. Wolfsthal. CoverageDirected Test Generation Using Symbolic Techniques. In First Int. Conf. on Formal Method in Computer-Aided Design (FMCAD), LNCS 1166, 1996, pp. 143158. 260 
[9] M. Glusman, G. Kamhi, S. Mador-Heim, R. Fraer, M. Vardi. Multiple-Counterexample Guided Iterative Abstraction Refinement: An Industrial Evaluation. In Tools and Algorithms for the construction and analysis of syst. (TACAS), LNCS 2619, 2003, pp. 176-191. 272

[10] A. Gurfinkel, M. Chechik. Proof-Like Counter-Examples. In Tools and Algorithms for the construction and analysis of syst. (TACAS), LNCS 2619, 2003, pp. 160175. 259, 262, 272

[11] P. H. Ho, T. Shiple, K. Harer, J. Kukula, R. Damiano, V. Bertacco, J. Taylor, J. Long. Smart Simulation Using Collaborative Formal and Simulation Engines. In Int. Conf. on Computer Aided Design (ICCAD), 2000. 260

[12] M. Maidl. The Common Fragment of CTL and LTL. In Proc. 41th Symp. on Foundations of Computer Science (FOCS), pp. 643-652, 2000. 259

[13] R. De Nicola, F. W. Vaandrager. Actions versus State Based Logics for Transition Systems. Proc. Ecole de Printemps on Semantics of Concurrency, Lecture Notes in Computer Science, vol. 469, 1990, pp. 407-419. 260, 262

[14] G. Ratzaby, S. Ur, Y. Wolfsthal, Coverability Analysis Using Symbolic Model Checking. In Conf. On Correct Hardware Design and Verification Methods (CHARME), LNCS 2144, 2001. 260

[15] G. Ratsaby, B. Sterin, S. Ur. Improvements in Coverabiliy Analysis. In Int. Symp. of Formal Methods Europe (FME), LNCS 2391, 2002. 260 


\section{Appendix}

NOTE: Automata in dashed polygon are obtained by a minimization.

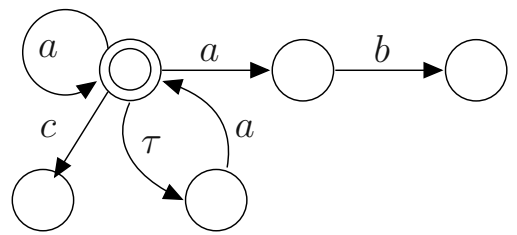

(a) The model: $S=a . S+\tau . a . S+a . b . n i l+c . n i l$

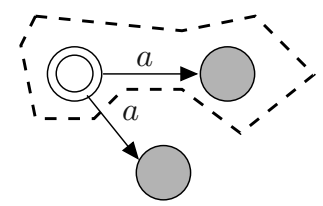

(b) Witness automaton for $\exists \mathbf{X}_{a}$ true

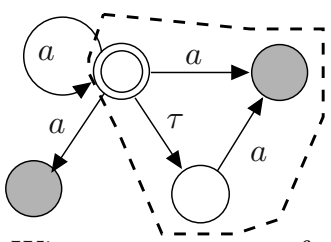

(d) Witness automaton for

$\exists \mathbf{F} \exists \mathbf{X}_{a}$ true

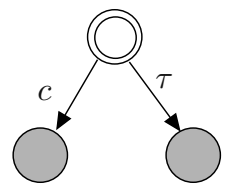

(f) Counterexample automaton for $\forall \mathbf{X}_{a}$ true

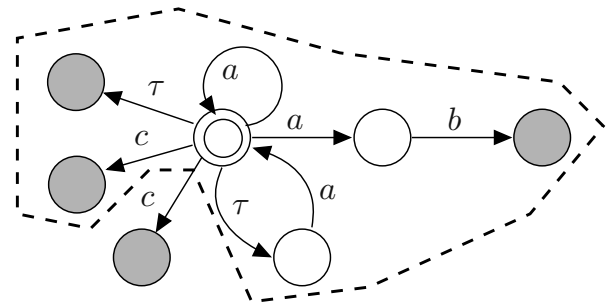

(h) Counterexample automaton for

$\forall \mathbf{G} \forall \mathbf{X}_{a}$ true

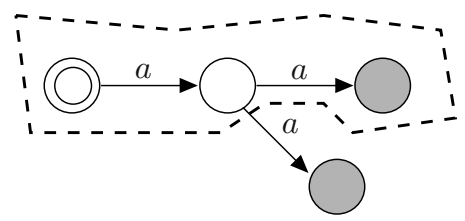

(c) Witness automaton for $\exists \mathbf{X}_{a} \exists \mathbf{X}_{a}$ true

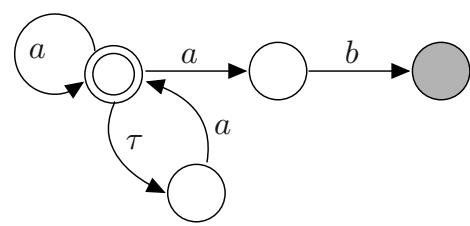

(e) Witness automaton for $\exists\left(\right.$ true $_{a} \mathbf{U}_{b}$ true $)$

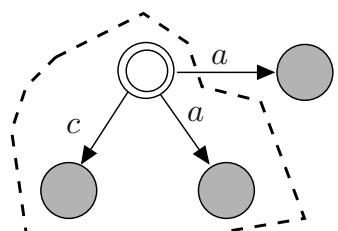

(g) Counterexample automaton for $\forall \mathbf{X}_{\tau} \forall \mathbf{X}_{a}$ true

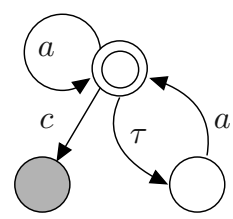

(i) An automaton generated for $\forall\left(\right.$ true $_{a} \mathbf{U}_{b}$ true $)$ 